\title{
Cauchy Transforms of Self-Similar Measures
}

\author{
John-Peter Lund, Robert S. Strichartz, and Jade P. Vinson
}

\section{CONTENTS}

\section{Introduction}

2. The Laurent Expansion

3. The Algorithm

4. The Sierpiński Gasket

Acknowledgments

References
Lund's and Vinson's research was supported by the National Science Foundation through the Research Experiences for Undergraduates (REU) Program.

Strichartz's research was supported in part by the National Science Foundation, grant DMS-9623250.
The Cauchy transform of a measure in the plane,

$$
F(z)=\frac{1}{2 \pi i} \int_{\mathbb{C}} \frac{1}{z-w} d \mu(w),
$$

is a useful tool for numerical studies of the measure, since the measure of any reasonable set may be obtained as the line integral of $F$ around the boundary. We give an effective algorithm for computing $\mathrm{F}$ when $\mu$ is a self-similar measure, based on a Laurent expansion of $F$ for large $z$ and a transformation law (Theorem 2.2) for $\mathrm{F}$ that encodes the self-similarity of $\mu$. Using this algorithm we compute $F$ for the normalized Hausdorff measure on the Sierpiński gasket. Based on this experimental evidence, we formulate three conjectures concerning the mapping properties of $\mathrm{F}$, which is a continuous function holomorphic on each component of the complement of the gasket.

\section{INTRODUCTION}

Let $\mu$ be a probability measure in the complex plane $\mathbb{C}$. The Cauchy transform of $\mu$ is defined formally by

$$
F(z)=\frac{1}{2 \pi i} \int_{\mathbb{C}} \frac{1}{z-w} d \mu(w)
$$

(see [Mattila 1995]). Note that $F$ is always welldefined and holomorphic in the complement of the support of $\mu$. For many measures we can meaningfully extend (1-1) to the whole plane, and $F$ will allow us to effectively recover the measure $\mu$ via the formula

$$
\mu(U)=\int_{\partial U} F(z) d z
$$

(where the contour is oriented counterclockwise) for any reasonable set $U$ - say with piecewise $C^{1}$ boundary. Indeed, (1-2) follows from (1-1) and the Cauchy integral formula as long as we can justify the interchange of integrals. This suggests that 
the Cauchy transform might be an effective tool for numerical analysis of measures in the plane.

In this paper we will be concerned with selfsimilar measures $\mu$. These are solutions of selfsimilar identities

$$
\mu=\sum_{j=1}^{m} p_{j} \mu \circ S_{j}^{-1},
$$

where $S_{j}$ are contractive similarities (forming what is called an iterated function system, or i.f.s.) and $p_{j}$ are probabilities ( $s o \sum p_{j}=1$ and $p_{j} \geq 0$ for all $j$ ). A well-known theorem of Hutchinson [1981] asserts that there is a unique probability measure satisfying (1-3), supported on the compact set $K$ which is the attractor of the i.f.s. (the unique compact set satisfying $\left.K=\bigcup_{j=1}^{m} S_{j} K\right)$. We will usually assume that the i.f.s. satisfies the open set condition: there exists an open set $U$ such that $S_{j} U \subseteq U$ and the sets $S_{j} U$ are disjoint, which implies the measure separation condition [Schief 1994]

$$
\mu\left(\overline{S_{j} U} \cap \overline{S_{k} U}\right)=0 \text { for } j \neq k .
$$

It was suggested in [Strichartz et al. 1995] that self-similar identities (1-3) play a role for measures analogous to differential equations for functions. Part of this analogy is that there exist numerical methods to compute approximations to the solution of self-similar identities (1-3). This is a relatively straightforward problem when the underlying space is the line [Strichartz et al. 1995], but is a more challenging problem even for the plane. In this paper we show that there exist effective algorithms for approximating the Cauchy transform of self-similar measures; together with (1-2) this gives one approach to the numerical approximation of the measure. One of the weaknesses of this approach is the difficulty of performing the numerical integrations required in (1-2): since the function $F$ is not particularly smooth, the usual methods of numerical integration cannot be expected to perform well.

We note that the reconstruction formula (1-2) is redundant, since the integral on the right side is complex valued, while the left side is a nonnegative real. This is not necessarily such a bad thing, since in numerical approximations the size of the imaginary part of the approximation to the integral might be a good indicator of the size of the error. It is easy to eliminate this redundancy, and complex numbers, by considering the pair of real functions

$$
\begin{aligned}
& F_{1}(x, y)=\frac{1}{2 \pi} \int_{\mathbb{R}^{2}} \frac{x-x^{\prime}}{\left(x-x^{\prime}\right)^{2}+\left(y-y^{\prime}\right)^{2}} d \mu\left(x^{\prime}, y^{\prime}\right), \\
& F_{2}(x, y)=\frac{1}{2 \pi} \int_{\mathbb{R}^{2}} \frac{y-y^{\prime}}{\left(x-x^{\prime}\right)^{2}+\left(y-y^{\prime}\right)^{2}} d \mu\left(x^{\prime}, y^{\prime}\right),
\end{aligned}
$$

so that

$$
F=-F_{2}-i F_{1}
$$

and (1-2) becomes

$$
\mu(U)=\int_{\partial U}\left(-F_{2} d x+F_{1} d y\right) .
$$

This approach generalizes easily to higher-dimensional spaces, but we will not pursue it in this paper since we are especially interested in the properties of the Cauchy transform that are connected with complex analysis.

Another perspective on the Cauchy transform is that it provides a solution of the differential equation

$$
\frac{\partial}{\partial \bar{z}} F=\mu
$$

and of course any solution of this equation differs from the Cauchy transform by an entire holomorphic function. In this way, the Cauchy transform is a complex analog of the potentials of classical potential theory. We will not use this observation in our work, however.

We now outline the contents of this paper. In Section 2 we discuss some elementary properties of the Cauchy transform. We show that the hypothesis

$$
p_{j}<r_{j} \quad \text { for all } j,
$$

where $r_{j}$ is the contraction ratio of the similarity $S_{j}$, suffices to make $F$ a continuous function. In fact (1-5) implies that $\mu$ has a uniform dimension 
greater than 1 , and this is the condition that implies continuity. We also show that $F$ satisfies a transformation law (see (2-7)) that is reminiscent of the transformation law for the Fourier transform of $\mu$ [Strichartz 1990]. We then show how to compute the Laurent expansion of $F$ in the complement of a disk containing $K$. In Section 3 we give an algorithm for the approximate computation of $F(z)$ by combining the transformation law and the Laurent expansion. We show that the number of operations required to guarantee an error of at most $\varepsilon$ is $O\left(|\log \varepsilon|^{2}\right)$.

In Section 4 we turn our attention to a special case, when $\mu$ is the normalized Hausdorff measure on the Sierpiński gasket. We display the data obtained by carrying out the algorithm, and describe three conjectures that are suggested by the data. The first two concern the mapping properties of $F$. It appears that the image of the plane under $F$ is the region inside of a certain curve, which is itself the image under $F$ of a Cantor set contained in the boundary triangle of the Sierpiński gasket. It is easy to see that the exterior of this triangle is mapped to the interior of the curve, but there is no apparent explanation for why the interior of the triangle should also end up mapped to the same region. We are able to prove only that a neighborhood of the midpoints of the edges of the triangle are mapped to this region. The third conjecture concerns the behavior of $F$ in a neighborhood of a vertex of the triangle. Of course $F$ is holomorphic in the exterior of the triangle. The data suggest that $F$ follows an approximate fractional power law in this sector. We have only numerical evidence for this behavior.

\section{THE LAURENT EXPANSION}

For simplicity, we consider only the case of orientation-preserving similarities, so we can write

$$
S_{j} z=z_{j}+r_{j} e^{i \theta_{j}}\left(z-z_{j}\right),
$$

where $z_{j}$ is the fixed point of $S_{j}$ and $0<r_{j}<1$ is the contraction ratio. We may assume without loss of generality that $r_{1} \geq r_{2} \geq r_{3} \geq \cdots$. If $r_{1}$ is close to 1 , the i.f.s. converges at a slow rate and our algorithms will perform poorly.

The assumption we need to make on the selfsimilar measure, in addition to the open set condition with measure separation (1-4), is

$$
p_{j}<r_{j} \quad \text { for all } j \text {. }
$$

As we will see, this forces $\mu$ to have dimension greater than one in the uniform sense that

$$
\mu(A) \leq c \operatorname{diam}(A)^{\alpha}
$$

for some $c$ and $\alpha>1$.

Theorem 2.1. (a) If $\mu$ is a self-similar measure satisfying the open set condition and condition (2-2), then $\mu$ satisfies (2-3) with $\alpha>1$, namely

$$
\alpha=\min \left\{\log p_{j} / \log r_{j}\right\}
$$

(b) If $\mu$ is any probability measure of compact support satisfying (2-3) with $\alpha>1$, then the Cauchy transform $F$ is a well-defined bounded continuous function on $\mathbb{C}$, satisfying a uniform Hölder estimate

$$
\left|F\left(z_{1}\right)-F\left(z_{2}\right)\right| \leq c\left|z_{1}-z_{2}\right|^{\alpha-1}
$$

of order $\alpha-1$ (for $\alpha<2)$. In particular, the integral (1-1) is absolutely convergent and (1-2) holds.

Proof. (a) For any multiindex $J=\left(j_{1}, \ldots, j_{N}\right)$ write $S_{J}=S_{j_{1}} \circ S_{j_{2}} \circ \cdots \circ S_{j_{N}}, r_{J}=r_{j_{1}} r_{j_{2}} \cdots r_{j_{N}}$, and $p_{J}=p_{j_{1}} p_{j_{2}} \cdots p_{j_{N}}$. Let $U$ be the open set in the open set condition. Then $\mu\left(S_{J} U\right)=p_{J}$, by the measure separation condition (1-4). A standard stopping time argument yields uniform constants $M, c_{1}$, and $c_{2}$ such that any set $A$ of diameter $d$ can be partitioned into at most $M$ sets of the form $A \cap S_{J} U$ with $c_{1} d \leq r_{J} \leq c_{2} d$ (in the sense that $\left.\mu(A)=\sum \mu\left(A \cap S_{J} U\right)\right)$. Since $p_{J} \leq r_{J}^{\alpha}$ by $(2-4)$, we have $\mu(A) \leq \sum p_{J} \leq \sum r_{J}^{\alpha} \leq M c_{2}^{\alpha} d^{\alpha}$, which is $(2-3)$. 
(b) Let $\mu$ be supported in a disk of radius $R$. Then all statements are straightforward for points outside the disk of radius $2 R$. Now $(2-3)$ in particular implies that $\mu$ is nonatomic, so we may partition the integral (1-1) into annular regions $A_{k}=\left\{w: 2^{k} \leq|w-z| \leq 2^{k+1}\right\}$ for $-\infty<k \leq k_{0}$. By $(2-3)$ we have $\mu\left(A_{k}\right) \leq c 2^{k \alpha}$ and of course $|w-z|^{-1} \leq c 2^{-k}$ on $A_{k}$. Thus

$$
\int_{A_{k}} \frac{d \mu(w)}{|z-w|} \leq c 2^{(\alpha-1) k}
$$

and since $\alpha>1$ this shows that (1-1) is an absolutely convergent integral, with a uniform bound. This is sufficient to justify the interchange of integrals

$$
\begin{aligned}
\int_{\partial U} F(z) d z & =\frac{1}{2 \pi i} \int_{\partial U} \int_{\mathbb{C}} \frac{d \mu(w)}{z-w} d z \\
& =\int_{\mathbb{C}}\left(\frac{1}{2 \pi i} \int_{\partial U} \frac{d z}{z-w}\right) d \mu(w) \\
& =\int_{U} d \mu(w)=\mu(U)
\end{aligned}
$$

to prove (1-2). To prove the Hölder estimate we fix $z_{1}, z_{2}$ distinct and let $\left|z_{1}-z_{2}\right|=d$. Then we can cover the support of $\mu$ by annular sets $A_{k}^{(1)}$ about $z_{1}$ and $A_{k}^{(2)}$ about $z_{2}$ with $2^{k} \leq 5 d / 2$ and sets $B_{k}$ obtained by deleting these sets from annular sets $A_{k}^{(3)}$ about the midpoint with $3 d \leq 2^{k}$. Now

$$
\begin{aligned}
\left|F\left(z_{1}\right)-F\left(z_{2}\right)\right| & =\left|\int_{\mathbb{C}}\left(\frac{1}{z_{1}-w}-\frac{1}{z_{2}-w}\right)\right| d \mu(w) \\
& \leq \int_{\mathbb{C}}\left|\frac{1}{z_{1}-w}-\frac{1}{z_{2}-w}\right| d \mu(w) .
\end{aligned}
$$

On $A_{k}^{(1)}$ and $A_{k}^{(2)}$ we use (2-6) and the crude estimate

$$
\left|\frac{1}{z_{1}-w}-\frac{1}{z_{2}-w}\right| \leq\left|\frac{1}{z_{1}-w}\right|+\left|\frac{1}{z_{2}-w}\right|
$$

to obtain $c\left(2^{k}\right)^{\alpha-1}$ for an upper bound which sums to $c d^{\alpha-1}$. On $B_{k}$ we use the estimate

$$
\left|\frac{1}{z_{1}-w}-\frac{1}{z_{2}-w}\right| \leq c d 2^{-2 k}
$$

to obtain $c d\left(2^{k}\right)^{\alpha-2}$ for an upper bound, which sums to $c d^{\alpha-1}$ since $\alpha<2$. This gives the Hölder estimate $(2-5)$, which proves in particular that $F$ is uniformly continuous.

Remark. Part (b) is essentially well-known [Carleson 1967; Garnett 1972, p. 78]. For related results see [Mattila and Melnikov 1994; Verdera 1992].

Theorem 2.2. Let $F$ be the Cauchy transform of a self-similar measure $\mu$. Then

$$
F(z)=\sum_{j=1}^{m} \frac{p_{j} e^{-i \theta_{j}}}{r_{j}} F\left(S_{j}^{-1} z\right) .
$$

Proof. From (1-1) and (1-3) we obtain

$$
F(z)=\frac{1}{2 \pi i} \sum_{j=1}^{m} p_{j} \int \frac{d \mu(w)}{z-S_{j} w} .
$$

But $z-S_{j} w=r_{j} e^{i \theta_{j}}\left(S_{j}^{-1} z-w\right)$.

Remark. If we allow some of the similarities to be orientation-reversing, of the form

$$
S_{j} z=z_{j}+r_{j} e^{i \theta_{j}}\left(\bar{z}-\bar{z}_{j}\right),
$$

then the only change in $(2-7)$ would be to replace $F\left(S_{j}^{-1} z\right)$ by $\overline{F\left(S_{j}^{-1} z\right)}$ for those $j$.

Let $D$ be the smallest closed disk containing $K$, the support of $\mu$. By translating the i.f.s., we may assume without loss of generality that $D$ is centered at the origin. Since $F$ is holomorphic in the complement of $D$ and vanishes at infinity, it has a Laurent expression with no nonnegative powers,

$$
F(z)=\sum_{n=1}^{\infty} a_{n} z^{-n} \quad \text { for } z \notin D .
$$

It is easy to see from $(1-1)$ that $a_{1}=1 /(2 \pi i)$. The other coefficients can be obtained recursively using $(2-7)$.

Theorem 2.3. The coefficients in the Laurent expansion satisfy

$$
\left(1-\sum_{j=1}^{m} p_{j}\left(r_{j} e^{i \theta_{j}}\right)^{n-1}\right) a_{n}=\sum_{k=1}^{n-1} B_{n, k} a_{k}
$$


for

$B_{n, k}=\left(\begin{array}{c}n-1 \\ n-k\end{array}\right) \sum_{j=1}^{m} p_{j}\left(r_{j} e^{i \theta_{j}}\right)^{k-1}\left(z_{j}\left(1-r_{j} e^{i \theta_{j}}\right)\right)^{n-k}$.

Proof. Substitute (2-8) in (2-7). Note that $S_{j}^{-1} z=$ $r_{j}^{-1} e^{-i \theta_{j}}\left(z-z_{j}\left(1-r_{j} e^{i \theta_{j}}\right)\right)$ so that

$$
\begin{aligned}
\left(S_{j}^{-1} z\right)^{-k}=( & \left.r_{j} e^{i \theta_{j}}\right)^{k} \\
& \times \sum_{l=0}^{\infty}\left(\begin{array}{c}
k-1+l \\
l
\end{array}\right) z^{-k-l}\left(z_{j}\left(1-r_{j} e^{i \theta_{j}}\right)\right)^{l}
\end{aligned}
$$

as a convergent Laurent series for $|z|$ sufficiently large. Thus we have an equality between the Laurent series (2-8) and

$$
\begin{aligned}
& \sum_{j=1}^{m} p_{j}\left(r_{j} e^{i \theta_{j}}\right)^{-1} \sum_{k=1}^{\infty}\left(a_{k}\left(r_{j} e^{i \theta_{j}}\right)^{k}\right. \\
&\left.\times \sum_{l=0}^{\infty}\left(\begin{array}{c}
k-1+l \\
l
\end{array}\right) z^{-k-l}\left(z_{j}\left(1-r_{j} e^{i \theta_{j}}\right)\right)^{l}\right) .
\end{aligned}
$$

Note that there are only a finite number of terms in this triple sum with the power $z^{-n}$, so that we may rearrange it into a Laurent expansion. Equating coefficients of the two expansions yields for $a_{n}$ the value

$$
\sum_{j=1}^{m} p_{j} \sum_{k=1}^{n} a_{k}\left(r_{j} e^{i \theta_{j}}\right)^{k-1}\left(\begin{array}{c}
n-1 \\
n-k
\end{array}\right)\left(z_{j}\left(1-r_{j} e^{i \theta_{j}}\right)\right)^{n-k} .
$$

We can transpose the $a_{n}$ term on the right (note that $\left.\left|\sum_{j=1}^{m} p_{j}\left(r_{j} e^{i \theta_{j}}\right)^{n-1}\right|<1\right)$ to the left to obtain (2-9) and (2-10).

Example 2.4. Let $\omega=-\frac{1}{2}+i \frac{\sqrt{3}}{2}$ be a cube root of unity and let

$$
S_{j} z=\frac{1}{2}\left(z+\omega^{j}\right) \text { for } j=0,1,2 .
$$

The attractor $K$ of this i.f.s. is just the familiar Sierpiński gasket (rotated from its usual presentation). If we choose $p_{j}=\frac{1}{3}$ then $\mu$ is just the normalized Hausdorff measure of dimension $\log 3 / \log 2$ on $K$. Because $K$ and $\mu$ are invariant under the $\frac{1}{3}$ rotation obtained by multiplication by $\omega$, it follows that the Cauchy transform $F$ satisfies

$$
F(z)=\omega F(\omega z)
$$

hence the only nonzero coefficients in the Laurent expansion $(2-8)$ are those with $n \equiv 1 \bmod 3$. The recursion relations $(2-9)$ and $(2-10)$ in this case simplify to

$$
a_{3 n+1}=\frac{1}{2^{3 n}-1} \sum_{k=0}^{n-1}\left(\begin{array}{c}
3 n \\
3(n-k)
\end{array}\right) a_{3 k+1} .
$$

Next we show that for many self-similar measures, including the above example, the Laurent expansion actually converges on the boundary circle of the disk $D$. For this we need the additional assumption that all $\theta_{j}=0$, so there are no rotations in the similarities $S_{j}$. This implies that $K$ is contained in the convex hull of the fixed points $z_{j}$. To simplify notation we assume that $D$ is the unit disk, so $\left|z_{j}\right| \leq 1$ for all $j$ with equality holding for some $j$.

Theorem 2.5. Under the above assumptions and the hypotheses of Theorem 2.1(a), for every $\beta<\alpha$ there exists c such that

$$
\left|a_{n}\right| \leq c n^{-\beta} .
$$

In particular, (2-8) converges uniformly on the unit circle.

Proof. For each $n$ and each $j$, we choose a positive integer $k_{j}$ so that $r_{j}^{k_{j}}$ is on the order of $n^{-\beta / \alpha}$. More precisely, we choose the smallest integer such that $r_{j}^{k_{j}} \leq n^{-\beta / \alpha}$, and then we have a uniform bound

$$
c n^{-\beta / \alpha} \leq r_{j}^{k_{j}} \leq n^{-\beta / \alpha} .
$$

Since we have $p_{j} \leq r_{j}^{\alpha}$ by $(2-4)$ we also know

$$
p_{j}^{k_{j}} \leq n^{-\beta}
$$

Now we remove from $K$ the sets $S_{j}^{k_{j}} K$, and the resulting set $K^{\prime}$ is contained in the disk of radius 
$1-c n^{-\beta / \alpha}$ by the lower bound in $(2-15)$. We use the formula

$$
a_{n}=\frac{1}{2 \pi i} \int_{D} w^{n-1} d \mu(w)
$$

for the coefficients of the Laurent expansion, which follows easily from (1-1). To estimate $a_{n}$ we break the integral up into the integrals over the sets $K^{\prime}$ and $S_{j}^{k_{j}} K$. For the first we have $|z| \leq 1-c n^{-\beta / \alpha}$ in $K^{\prime}$ and $\mu\left(K^{\prime}\right) \leq 1$. For the second we have $|z| \leq 1$ and $\mu\left(S_{j}^{k_{j}} K\right)=p_{j}^{k_{j}} \leq n^{-\beta}$ by $(2-16)$. Thus

$$
\left|a_{n}\right| \leq\left(1-c n^{-\beta / \alpha}\right)^{n-1}+m n^{-\beta}
$$

and since $\beta<\alpha$ we have $\left(1-c n^{-\beta / \alpha}\right)^{n} \leq c_{1} n^{-\beta}$ for a suitable choice of $c_{1}$ : just use the fact that $\log (1-x) \leq-x$ and choose $c_{1}$ so that $-c n^{1-\beta / \alpha} \leq$ $-\beta \log n+\log c_{1}$. This proves (2-14). Since $\alpha>1$ we can also choose $\beta>1$ to show the convergence of the Laurent expansion on the boundary circle.

\section{THE ALGORITHM}

We describe an algorithm for approximating $F(z)$ by combining $(2-7)$ and $(2-8)$. The idea is to iterate $(2-7)$ until the points are pushed outside $D$, and then evaluate $F$ at those points using the Laurent expansion. There are 3 parameters in the algorithm that influence both the accuracy and the number of computations required. The first parameter $R>1$ is an expansion factor for $D ; D^{\prime}$ is the disk with the same center as $D$ with radius multiplied by $R$. We will only evaluate the Laurent expansion at points outside $D^{\prime}$, which will guarantee rapid convergence. The second parameter $N$ is just the number of terms in the Laurent expansion that we take. The third parameter $\delta$ is a threshold; when the coefficient drops below $\delta$ we will discard the term. We always assume $\delta<1$.

By iterating $(2-7)$ we obtain

$$
F(z)=\sum_{J \in \mathscr{J}} \frac{p_{J}}{r_{J}} e^{-i \theta_{J}} F\left(S_{J}^{-1} z\right)
$$

where $\mathscr{J}$ is a finite collection of multiindices $J=$ $\left(j_{1}, \ldots, j_{k}\right)$. Here we use the notation

$$
\begin{aligned}
S_{J} & =S_{j_{1}} S_{j_{2}} \cdots S_{j_{k}}, \\
p_{J} & =p_{j_{1}} \cdots p_{j_{k}}, \\
r_{J} & =r_{j_{1}} \cdots r_{j_{k}}, \\
\theta_{J} & =\theta_{1}+\cdots+\theta_{k} .
\end{aligned}
$$

There are many different choices of collections $\mathscr{J}$ of multiindices for which $(3-1)$ is valid. For each $z$ the algorithm will construct the particular $\mathscr{J}$ by an iterative procedure, starting with

$$
\mathscr{J}=\{(1),(2), \ldots,(m)\} .
$$

At each iteration the multiindices $J$ are sorted into two groups, $\mathscr{J}^{\prime}$ and $\mathscr{J}^{\prime \prime}$. The elements of $\mathscr{J}^{\prime}$ are kept unchanged (and continue unchanged thereafter), while each $J$ in $\mathscr{J}^{\prime \prime}$ is replaced by the $m$ multiindices $(J, 1),(J, 2), \ldots,(J, m)$. The criterion for the selection is that $J$ goes in $\mathscr{J}^{\prime}$ if either $S_{J}^{-1} z$ is outside $D^{\prime}$ or $p_{J} / r_{J}$ drops below the threshold $\delta$. Because of assumption (2-2) the iteration eventually terminates. If

$$
\lambda=\max \left\{p_{j} / r_{j}\right\}
$$

then $p_{J} / r_{J} \leq \lambda^{k}$ if $J$ has length $k$, so $\log \delta / \log \lambda$ is an upper bound for the length of any $J$ in $\mathscr{J}$.

It might seem that the number of terms in the sum (3-1) would be quite large, but it turns out that it is quite modest, and all but a few multiindices $J$ are there because $S_{J}^{-1} z$ is outside $D^{\prime}$. To simplify the discussion we present first a complete analysis in the special case that all the contraction ratios $r_{j}$ are equal. We then briefly discuss the modifications needed to handle the general case.

Lemma 3.1. In the preceding special case, there exist constants $c_{1}, c_{2}$ depending on $R$ such that the number of terms in $(3-1)$ is at most $c_{1}|\log \delta|$, and the number for which $S_{J}^{-1} z$ is in $D^{\prime}$ is at most $c_{2}$.

Proof. Set $k_{0}=\lfloor\log \delta / \log \lambda\rfloor$, and for each $k \leq k_{0}$ let $\mathscr{J}_{k}$ denote the set of multiindices at stage $k$ of the construction, split into $\mathscr{J}_{k}^{\prime}$ and $\mathscr{J}_{k}^{\prime \prime}$ according to whether $S_{J}^{-1} z$ is outside $D^{\prime}$ or inside $D^{\prime}$. The 
key observation is that there is an upper bound $c_{2}$ for the number of elements of $\mathscr{J}_{k}^{\prime \prime}$. To see this observe that $S_{J}^{-1} z \in D^{\prime}$ is the same as $z \in S_{J} D^{\prime}$, and the disks $S_{J} D^{\prime}$ all have the same radius. Hence all $S_{J} D^{\prime}$ for $J \in \mathscr{J}_{k}^{\prime \prime}$ lie in the disk of radius $c R r^{k}$ about $z$, for $c$ equal to the diameter of $D$. On the other hand, the open set condition gives disjoint sets $S_{J} U$ lying inside the same disk. If $A$ is the area of $U$, then $S_{J} U$ has area $r^{2 k} A$, so $c_{1}=\pi c^{2} R^{2} / A$ is the upper bound for the cardinality of $\mathscr{J}_{k}^{\prime \prime}$. Finally, each $J$ in $\mathscr{J}_{k}^{\prime \prime}$ gives rise to $m$ multiindices in $\mathscr{J}_{k+1}$, so we have an upper bound of $c_{2} m k_{0}$ for the cardinality of $\mathscr{J}$, and this is of the form $c_{1}|\log \delta|$ for $c_{1}=c_{2} m /|\log \lambda|$.

The preceding argument made use of the fact that the sets $S_{J} D^{\prime}$ (and also $S_{J} U$ ) were of comparable size. In the general case (different $r_{j}$ ) we have to modify the way we break up the algorithm into steps $\mathscr{J}_{k}$ in order to achieve this. We choose the ordering so that $r_{1} \geq r_{1} \geq \cdots \geq r_{m}$. The multiindices $J$ in $\mathscr{J}_{k}$ will be chosen to satisfy $r_{m}^{k} \leq r_{J}<$ $r_{m}^{k-1}$. Specifically, $\mathscr{J}_{1}$ is just $\{(1),(2), \ldots,(m)\}$ as before, and each $J$ in $\mathscr{J}_{k}^{\prime \prime}$ is subdivided until $r_{J}$ first goes below $r_{m}^{k}$, to obtain the new elements of $\mathscr{J}_{k+1}$. There is still a uniform upper bound to the number of elements in $\mathscr{J}_{k+1}$ that arise from each $J$ in $\mathscr{J}_{k}^{\prime \prime}$, but it is larger than $m$. We again have an upper bound of $O(|\log \delta|)$ for the number of steps until all $p_{J} / r_{J}$ go below the threshold $\delta$. In this way the lemma can be established without the special assumptions.

Let $\mathscr{J}^{\prime}$ denote those multiindices in $(3-1)$ for which $S_{J}^{-1} z$ is outside $D^{\prime}$, and $\mathscr{J}^{\prime \prime}$ those for which $p_{J} / r_{J} \leq \delta$. To approximate $F(z)$ we discard the terms in (3-1) corresponding to $\mathscr{J}^{\prime \prime}$, and for the remaining terms we replace $F\left(S_{J}^{-1} z\right)$ by $N$ terms of the Laurent expansion (2-8). Specifically

$$
F(z) \approx \sum_{J \in \mathscr{J}^{\prime}} \frac{p_{J}}{r_{J}} e^{-i \theta_{J}} \sum_{n=1}^{N} a_{n}\left(S_{J}^{-1} z\right)^{-n} .
$$

The error in (3-2) due to dropping the $\mathscr{J}^{\prime \prime}$ terms is bounded by $M c_{2} \delta$, where $M=\|F\|_{\infty}$, since there are at most $c_{2}$ terms and the coefficient of each is bounded by $\delta$. The error in approximating $F$ by the Laurent expansion can be bounded by $\left(\frac{M_{1}}{R-1}\right) R^{-N}$, where $M_{1}=\sup \left|a_{n}\right|$. There are $c_{1}|\log \delta|$ such terms in (3-2), and the coefficients are a priori only bounded by 1 , so the bound for the error in $(3-2)$ is

$$
M c_{2} \delta+\left(\frac{c_{1} M_{1}}{R-1}\right)|\log \delta| R^{-N}
$$

We choose the parameters in our algorithm in the order $R, \delta, N$. A reasonable choice for $R$ is 2. This choice determines $c_{1}$ and $c_{2}$ and does not depend on the target error $\varepsilon$. We then choose $\delta=$ $\varepsilon / 2 M c_{2}$, so the first term in $(3-3)$ is $\varepsilon / 2$. Having chosen $\delta$, we choose $N$ large enough to bound the second term in (3-3) by $\varepsilon / 2$, which means $N$ is the smallest integer greater than or equal to

$$
\frac{\log \left(\frac{2 c_{1} M_{1}}{R-1}\right)+\log |\log \delta|+|\log \varepsilon|}{\log R}
$$

We can easily estimate the number of computations required to guarantee the target error $\varepsilon$. There are at most $c_{1}|\log \delta|$ terms in the $\mathscr{J}^{\prime}$ summation. Both $|\log \delta|$ and $N$ are on the order of $|\log \varepsilon|$. The transformation $S_{J}$, the ratio $p_{J} / r_{J}$, and the phase $\theta_{J}$ are are easily computed from the corresponding parameters for the parent multiindex of $J$. The most intensive part of the computation is the evaluation of a truncated Laurent series. For each $J$ in (3-2), this requires about $2 N$ operations. Thus the entire algorithm requires $O\left(|\log \varepsilon|^{2}\right)$ operations to compute $F(z)$ at a single point $z$ to within an error of $\varepsilon$.

This is a worst case analysis of the error and computational effort; usually the algorithm gives a better performance on both accounts. For points $z$ in the complement of $K$, all preimages $S_{J}^{-1} z$ are outside $D^{\prime}$ for $J$ of sufficient length. For this $z$ we can find a single $\mathscr{J}$ that works for all $\varepsilon$, and the only error arises from the truncation of the Laurent expansions.

Essentially the same thing is true if we take $z$ to be one of the fixed points $z_{j}$, but this requires a 
slight change in the algorithm. In this case $(2-7)$ has $F\left(z_{j}\right)$ on both sides of the identity. To simplify notation take $z=z_{1}$. Then (2-7) yields

$$
\left(1-\frac{p_{1} e^{-i \theta_{1}}}{r_{1}}\right) F\left(z_{1}\right)=\sum_{j=2}^{m} \frac{p_{j} e^{-i \theta_{j}}}{r_{j}} F\left(S_{j}^{-1} z_{1}\right)
$$

and the coefficient of $F\left(z_{1}\right)$ is nonzero because $p_{1}<$ $r_{1}$. We could use (3-5) in place of $(2-7)$ whenever $F\left(z_{1}\right)$ arises in one of the terms of the expansion in our algorithm. This will never occur for a generic point, but it occurs if we take for $z$ an image of a fixed point under $S_{J}$, and often these are the most prominent points in $K$.

Another modification that would yield a slight improvement in the algorithm would be not to discard all the $\mathscr{J}^{\prime \prime}$ terms, but to approximate $F\left(S_{J}^{-1} z\right)$ by taking $F\left(z^{\prime}\right)$ for $z^{\prime}$ a point near $S_{J}^{-1} z$. This would entail storing a table of values of $F$ at a grid of points in $D^{\prime}$, and then locating the nearest point to $z^{\prime}$ on the grid. The Hölder estimate $(2-5)$ controls the error involved in replacing $F\left(S_{J}^{-1} z\right)$ by $F\left(z^{\prime}\right)$, and this is multiplied by the small coefficient in $(3-1)$.

\section{THE SIERPIŃSKI GASKET}

We consider now the measure in Example 2.4 and its Cauchy transform. Let $T$ denote the triangle with vertices $1, \omega, \omega^{2}$. The Cauchy transform $F$ is holomorphic in the exterior of $T$, and also in each of the triangular components of the complement of the Sierpiński gasket $K$. Since the complement of $K$ is dense in the plane, it would suffice to understand the behavior of $F$ on each of these triangular domains. But this seems to be quite difficult. There is no canonical form for an analytic function in the interior or exterior of a triangle. Also, our data suggest that the behavior of $F$ becomes quite wild as $z$ approaches the boundary of a triangle.

We note that $(2-7)$, which now has the simple form

$$
F(z)=\frac{2}{3}\left(F(2 z-1)+F(2 z-\omega)+F\left(2 z-\omega^{2}\right)\right),
$$

enables us to connect the different holomorphic functions that $F$ becomes when restricted to these triangular domains in the complement of $K$. For example, if $z$ lies in the largest component, then each of the preimages $2 z-1,2 z-\omega, 2 z-\omega^{2}$ lies in the exterior of $T$. By iterating (4-1) we can obtain a formula for $F(z)$ on any component entirely in terms of $F\left(S_{J}^{-1} z\right)$ with $S_{J}^{-1} z$ in the exterior of $T$.

We implemented the algorithm described in Section 3 to compute $F$. Because this is a function from $\mathbb{C}$ to $\mathbb{C}$ it is impossible to display all the data graphically. In Figure 1 we show the graph of the restriction of $\operatorname{Im} F$ to the $x$-axis ( $\operatorname{Re} F$ is zero there). The $x$-axis passes through a countable sequence of triangular components of the complement of $K$, accumulating at the point $x=1$, and the graph shows that the holomorphic pieces join together in what appear to be cusp singularities. In Figure 2 we show the graph of the restrictions of Re $F$ and $\operatorname{Im} F$ to one side of the triangle $T$ (rescaled to the unit interval). These functions seem to exhibit fractal behavior. In Figure 3 we show the image of the triangle $T$ under $F$. This is a curve with many self-intersections. Let $A$ denote the unbounded component of the complement of $F(T)$, and $B$ the complement of $A$. We think of $A$ as the "exterior" of $F(T)$, and the interior of $B$ as the "interior" of $F(T)$.

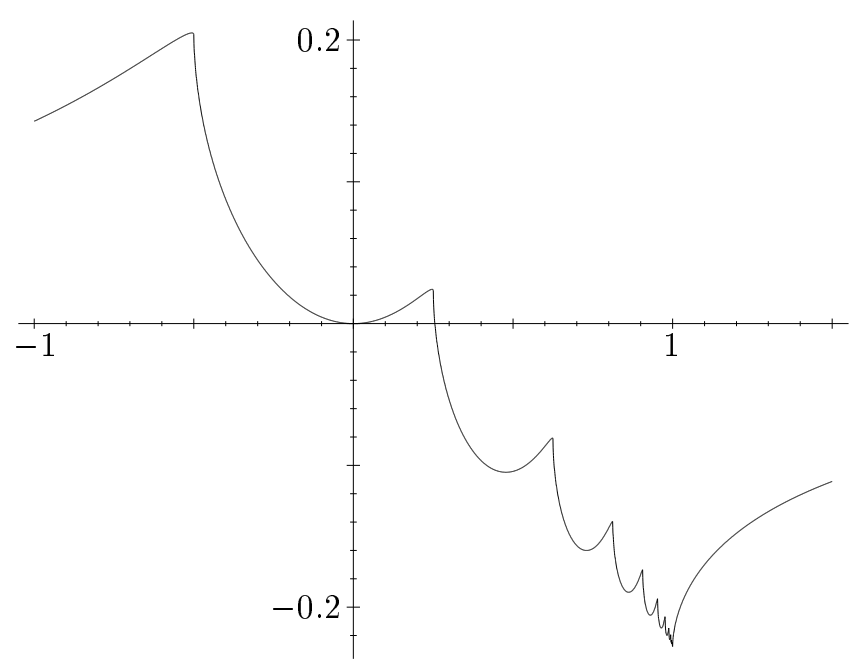

FIGURE 1. The restriction of $\operatorname{Im} F$ to the $x$-axis. 


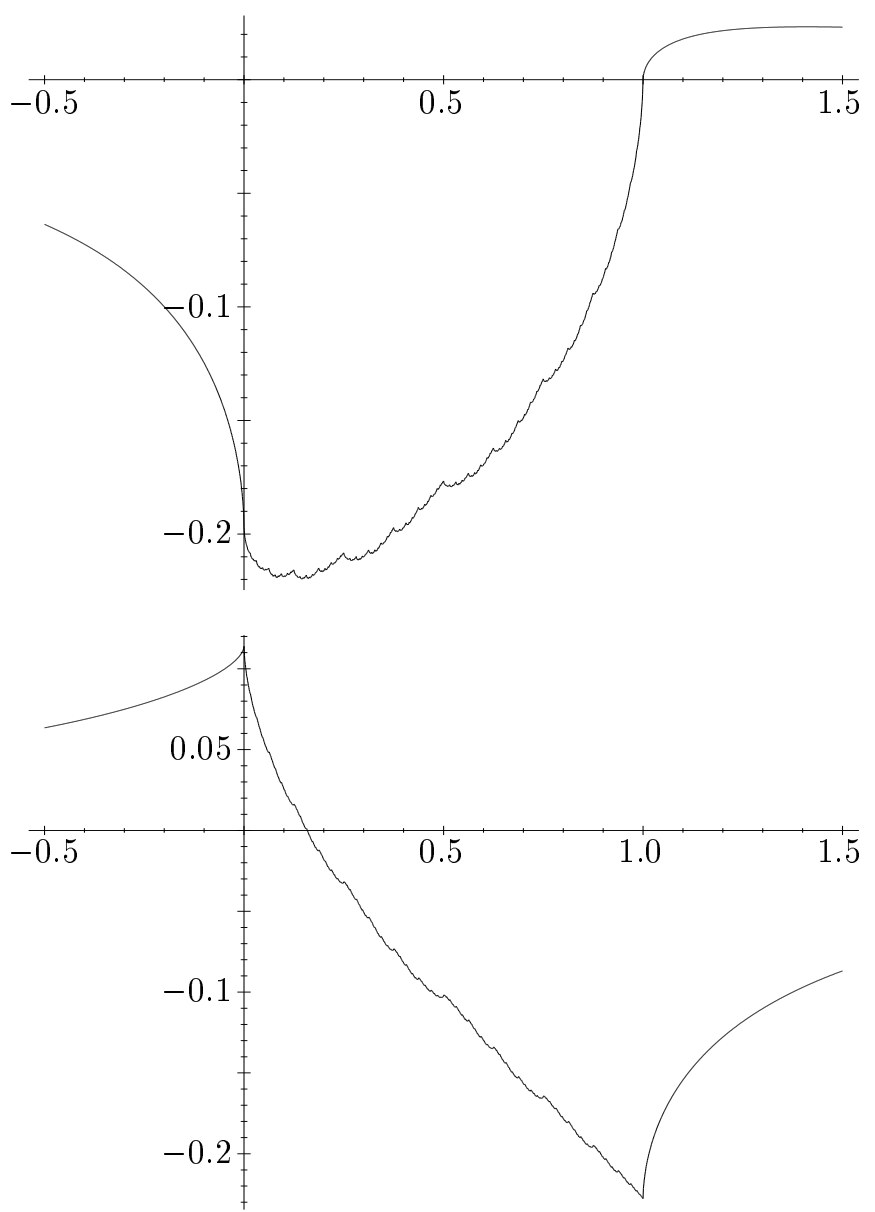

FIGURE 2. The restriction of $\operatorname{Re} F$ (top) and $\operatorname{Im} F$ (bottom) to the line $\omega+t(1-\omega)$ through the vertices $\omega=\left(-\frac{1}{2}+i \frac{\sqrt{3}}{2}\right)$ and 1 of the triangle $T$.

Conjecture 4.1. The interior of $B$ is connected, and the boundary of $B$, which we denote by $\Gamma$, is a simple closed curve, the image of a Cantor set $C$ in $T$ (the complement of $C$ in $T$ is a dense countable union of open intervals).

The conjecture in particular says that $F(T)$ has self-intersections at the images of the endpoints of the intervals in the complement of $C$, and then loops into the interior of $B$ on the interval (the loop must go in rather than out for the interior of $B$ to be connected). We are not asserting that these are the only self-intersections of $F(T)$; in fact the data seems to show self-intersection in the image of the

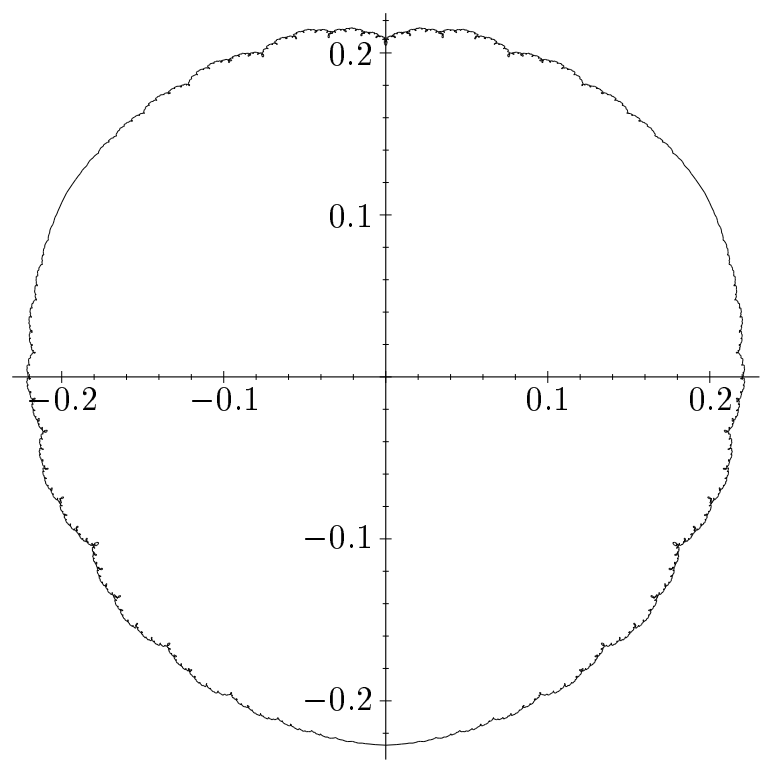

FIGURE 3. The image of the triangle $T$ under the mapping $F$.

intervals in the complement of $C$.

It follows from the open mapping property of analytic functions that the exterior of $T$ gets mapped to the interior of $B$. Figure 4 shows the image of the 27 triangles obtained from $T$ after 3 iterations of the i.f.s.

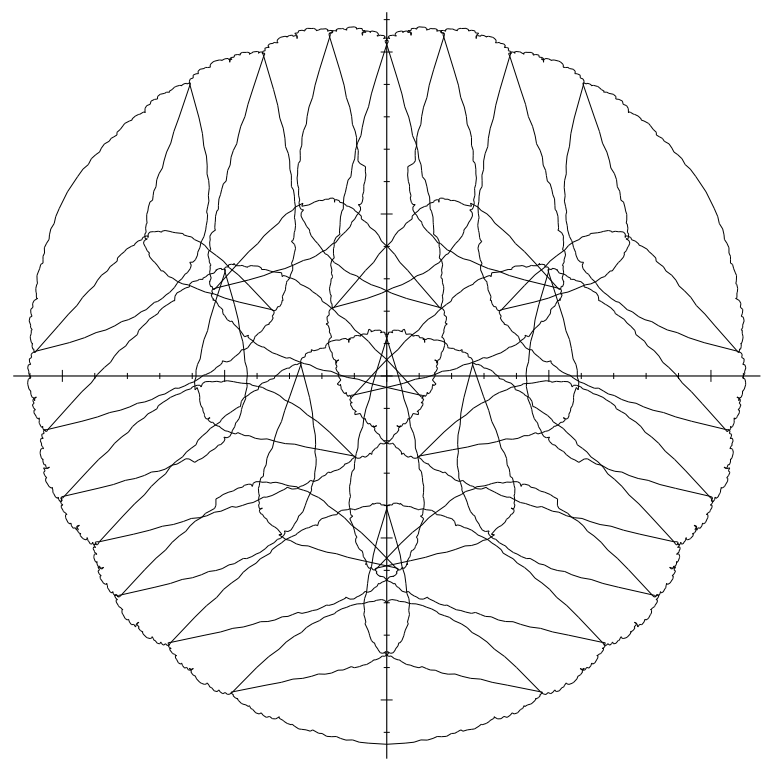

FIGURE 4. The image under $F$ of the 27 triangles obtained from $T$ after three iterations of the i.f.s. 
Conjecture 4.2. The image of $\mathbb{C}$ under $F$ is exactly $B$, and $F(z)$ lies in the interior of $B$ unless $z$ is in T. Also $F(K)$ is dense in $B$.

We present a small piece of evidence for these conjectures by analyzing the behavior of $F(z)$ near a midpoint of a side of $T$. Because of $(2-12)$ there is complete symmetry, so it suffices to deal with the point $-\frac{1}{2}$.

Theorem 4.3. $F\left(-\frac{1}{2}\right)$ lies in the interior of $B$.

Proof. We look at $f(t)=-2 \pi i F\left(-\frac{1}{2}-t\right)$ for $t \geq 0$. This is a real-valued function because of symmetry of $\mu$ under $z \rightarrow \bar{z}$; in fact

$$
F(x)=\frac{1}{4 \pi i} \int\left(\frac{1}{x-w}+\frac{1}{x-\bar{w}}\right) d \mu(w)
$$

for $x$ real, so

$$
f(t)=\int \frac{t+u+\frac{1}{2}}{\left(t+u+\frac{1}{2}\right)^{2}+v^{2}} d \mu(w),
$$

where $w=u+i v$. Note that $f(t)$ is positive, and $f(t) \rightarrow 0$ as $t \rightarrow \infty$. We claim that $f(t)$ is actually increasing for $t$ near 0 . If we can prove this we are done, because that would mean there exists $t_{0}>0$ with $f\left(t_{0}\right)=f(0)$, hence $F\left(-\frac{1}{2}\right)=F\left(-\frac{1}{2}-t_{0}\right)$. Since $-\frac{1}{2}-t_{0}$ lies outside $T, F\left(-\frac{1}{2}-t_{0}\right)$ must lie in the interior of $B$.

To show $f$ is increasing we need to show $f^{\prime}>0$. We will actually prove $f^{\prime}(0)=+\infty$, which makes $f^{\prime}(t)>0$ for $t$ near 0 . We can differentiate $(4-2)$ for $t>0$ to obtain

$$
f^{\prime}(t)=\int \frac{v^{2}-\left(\frac{1}{2}+u+t\right)^{2}}{\left(v^{2}+\left(\frac{1}{2}+u+t\right)^{2}\right)^{2}} d \mu(w) .
$$

We claim that this is a divergent integral for $t=0$. To see this we consider how this integral behaves under the contraction $S z=\frac{1}{2}\left(z-\frac{1}{2}\right)$ with fixed point $-\frac{1}{2}$ (this is not a mapping in the i.f.s.). The measure is multiplied by $\frac{1}{3}$, while the integrand is multiplied by $\frac{4}{3}$. Let $T_{1}=S_{1} S_{1} T$ and $T_{2}=S_{1} S_{0} T$, so that $S_{1} T$ is the disjoint union of $S^{k} T_{1}$ and $S^{k} T_{2}$ for $k \geq 0$. Then

$$
\begin{aligned}
& \int_{S^{k} T_{1}} \frac{v^{2}-\left(\frac{1}{2}+u\right)^{2}}{\left(v^{2}+\left(\frac{1}{2}+u\right)^{2}\right)^{2}} d \mu(w) \\
& =\left(\frac{4}{3}\right)^{k} \int_{T_{1}} \frac{v^{2}-\left(\frac{1}{2}+u\right)^{2}}{\left(v^{2}+\left(\frac{1}{2}+u\right)^{2}\right)^{2}} d \mu(w) .
\end{aligned}
$$

Since the integrand is positive on $T_{1}$, we conclude that the integral in (4-3) diverges at $t=0$.

Now the contribution to the integral from the triangle $S_{0} T$ is finite, so we can ignore it, and by symmetry the contributions from $S_{1} T$ and $S_{2} T$ are equal. To show that $f^{\prime}(0)=+\infty$ it suffices to show that the integral over $T_{1} \cup T_{2}$ is positive. This is not quite as simple as before since the integrand is negative on a portion of $T_{2}$. But consider the reflection symmetry about the line joining $-\frac{1}{2}$ to the common vertex of $T_{1}$ and $T_{2}$. This interchanges $T_{1}$ and $T_{2}$, leaves the measure invariant, and also leaves the denominator $\left(v^{2}+\left(u+\frac{1}{2}\right)^{2}\right)^{2}$ unchanged. Finally, we need to see that the sum of the numerator at two symmetric points is positive. These points must be of the form

$$
(u, v)=\left(-\frac{1}{2}+\lambda\left(\frac{3}{8} \pm s\right), \lambda\left(\frac{3 \sqrt{3}}{8} \mp s \frac{\sqrt{3}}{3}\right)\right),
$$

and the sum of $v^{2}-\left(\frac{1}{2}+u\right)^{2}$ is equal to

$$
\lambda^{2}\left(\frac{9}{32}-\frac{2}{3} s^{2}\right) .
$$

Also $|s| \leq \frac{3}{8}$ for points in $T_{1}$ or $T_{2}$, so $(4-4)$ is positive.

This shows that for $t=0$ the integral in (4-3) diverges to $+\infty$. Of course this argument also shows that if we take $t>0$ close to 0 then (4-3) will be positive.

We illustrate this behavior in Figure 5, using the "dartboard" method. To visualize the mapping $F$ in a neigbborhood of a point we display the image under $F$ of a dartboard (or portion of a dartboard) centered at the point, meaning a set of equally spaced radial spokes and concentric circles of constant polar coordinates. In Figure 5, top, we use 


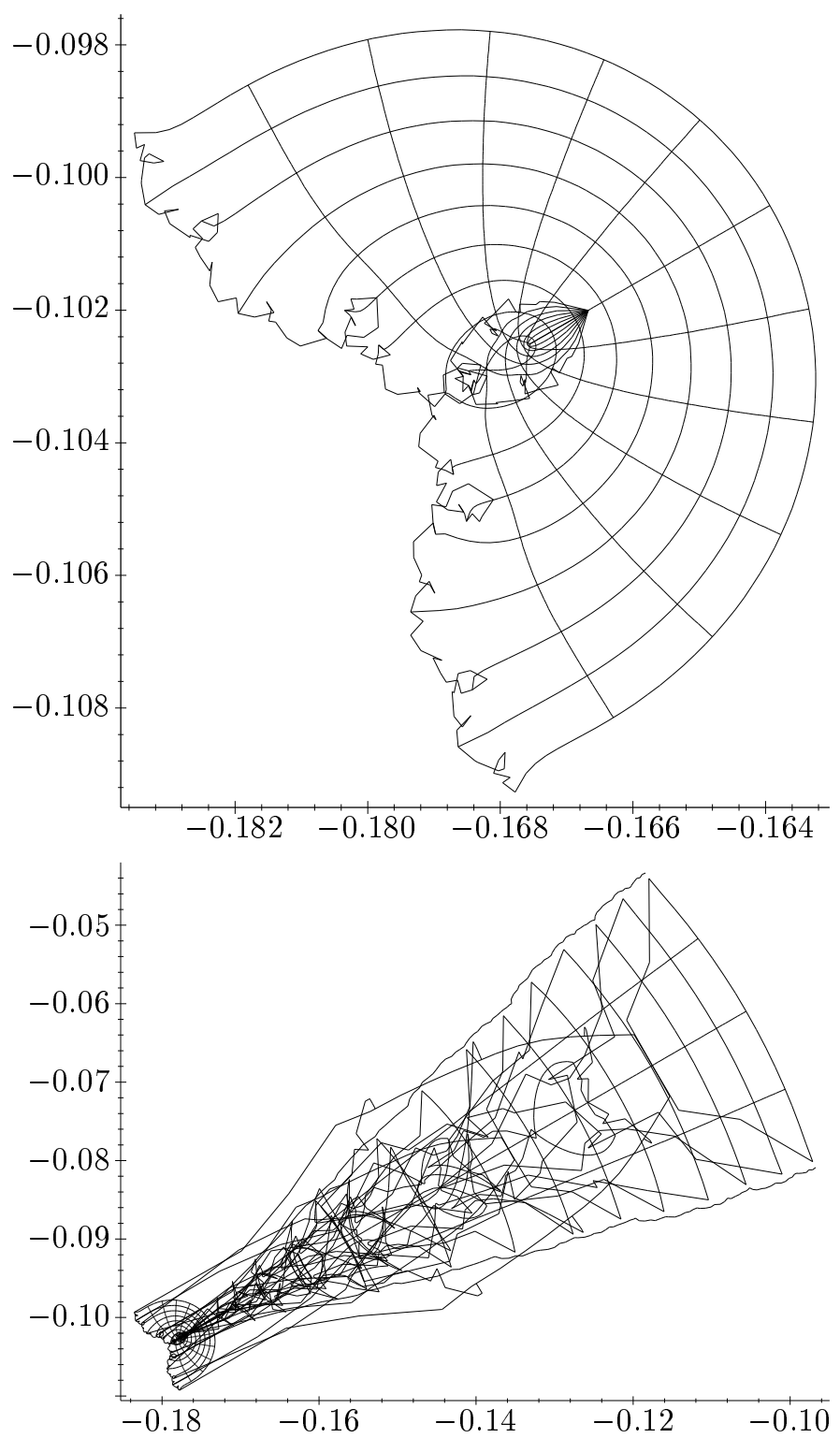

FIGURE 5. Top: The image under the mapping $F$ of a half dartboard centered at the midpoint of a side of $T$ (in this case $\frac{1}{2}(1+\omega)$ ) and extending into the exterior of $T$. Bottom: The same for the full dartboard, shown to a different scale.

the half of a dartboard exterior to $T$ at a midpoint (in this case $\frac{1}{2}(1+\omega)$ ) of a side. The image of the midpoint lies at the tip of the loop that goes inside $B$. For comparison, we show in Figure 5 , bottom, the image of the whole dartboard. Note that the behavior of $F$ in the interior of $T$ is much more complicated, but the picture is consistent with Conjecture 4.2.

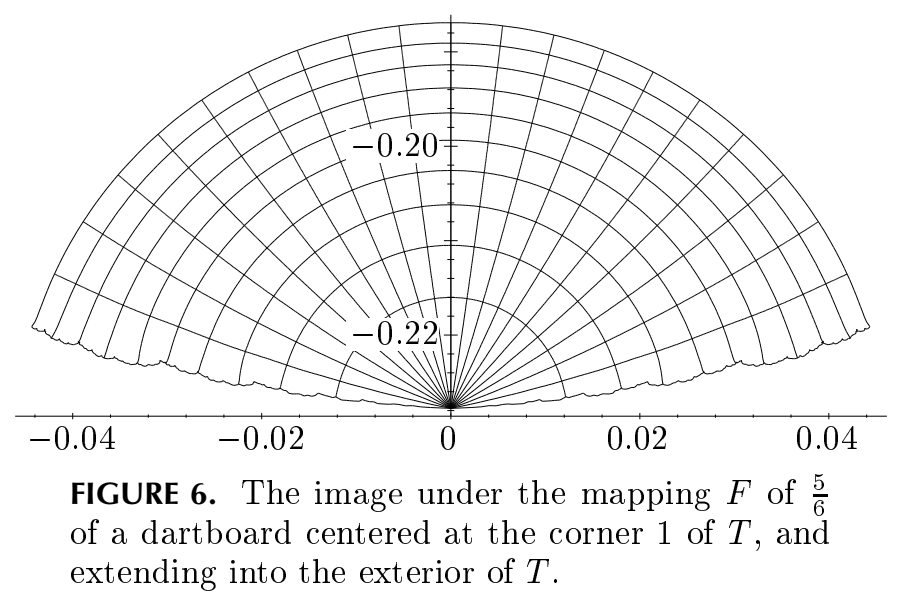

In Figure 6 we show the image of the exterior portion of a dartboard centered at the corner 1 (the angular variable is restricted to $\left.-\frac{5}{6} \pi \leq \theta \leq \frac{5}{6} \pi\right)$. The image of the boundary spokes $\theta= \pm \frac{5}{6} \pi$ follows the boundary curve $\Gamma$ (with loops into $B$ ), but away from these spokes the image suggests an approximate power law. Simple heuristic considerations lead to the power $\alpha-1$, where $\alpha=\log 3 / \log 2$ is the dimension of $K$, and this is confirmed by the data.

Conjecture 4.4. There exists an imaginary constant $b$ such that

$$
F(z)=F(1)+b(z-1)^{\alpha-1}+O(|z-1|)
$$

holds in any sector

$$
-\frac{5}{6} \pi+\varepsilon \leq \operatorname{Arg}(z-1) \leq \frac{5}{6} \pi-\varepsilon
$$

for $\varepsilon>0$.

As evidence for this conjecture we attempted to estimate the constant $b$ by fitting the data to (4-5), and in fact we guessed a simple linear term for the $O(|z-1|)$ remainder,

$$
F(z) \approx F(1)+b(z-1)^{\alpha-1}+c(z-1) .
$$

By trial and error we found that $b=0.2764 i$ and $c=-0.1421 i$ give very good agreement in $(4-7)$. 

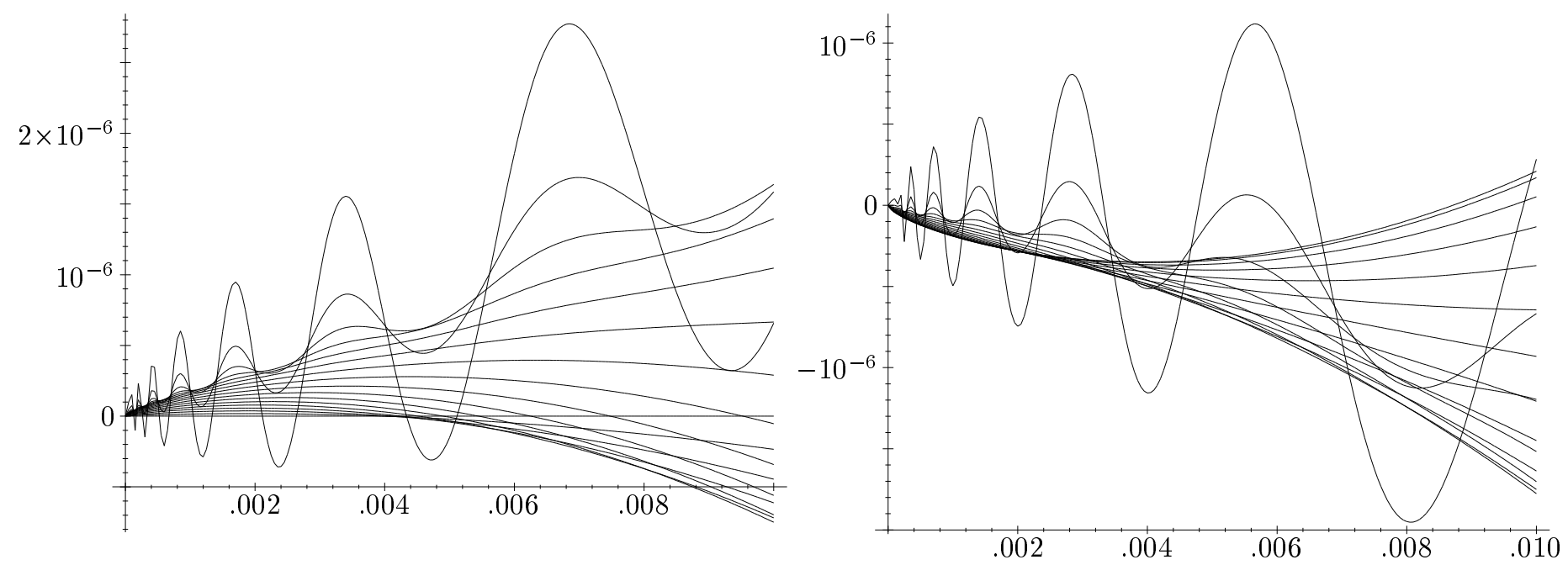

FIGURE 7. The real (left) and imaginary (right) parts of the difference between $F(z)$ and the approximation $F(1)+0.2764 i(z-1)^{\alpha-1}-0.1421 i(z-1)$. Each line is the restriction to one of the radial spokes of the dartboard, for $-\frac{2}{3} \pi \leq \theta \leq \frac{2}{3} \pi$.

In Figure 7 we show the real and imaginary parts of the difference between the two sides in (4-7) along the radial spokes of the dartboard corresponding to $-\frac{2}{3} \pi \leq \theta \leq \frac{2}{3} \pi$. In fact it seems likely that $(4-5)$ is only the first term of a complete asymptotic expansion.

We conclude by showing some variants of Figure 6 for different i.f.s. We change the contraction ratio from $\frac{1}{2}$ to obtain the i.f.s.

$$
S_{j} z=r z+(1-r) w^{j} \quad \text { for } j=0,1,2,
$$

where $r$ is the new contraction ratio, and take $\mu$ to be unique probability measure satisfying (1-3) with $p_{1}=p_{2}=p_{3}=\frac{1}{3}$. For $r>\frac{1}{2}$ the images
$S_{j} K$ overlap, so $\mu$ is not the normalized Hausdorff measure on $K$ (in fact, the Hausdorff dimension of $K$ is not known), although $K$ is connected and lies inside $T$. For $r<\frac{1}{2}, K$ is totally disconnected and $\mu$ is the normalized Hausdorff measure of dimension $\alpha=\log 3 / \log (1 / r)$. We display the analogous image to Figure 6 for the choices $r=0.51$ and $r=0.49$ in Figure 8. The overall pattern is similar, but the behavior of the image of the boundary spokes changes significantly.

If we replace the three third roots of unity with the five fifth roots of unity, and choose the contraction ratio $\tau^{-2}$ (where $\tau=\frac{1}{2}(\sqrt{5}+1) / 2$, the golden ratio), then we obtain the self-similar set
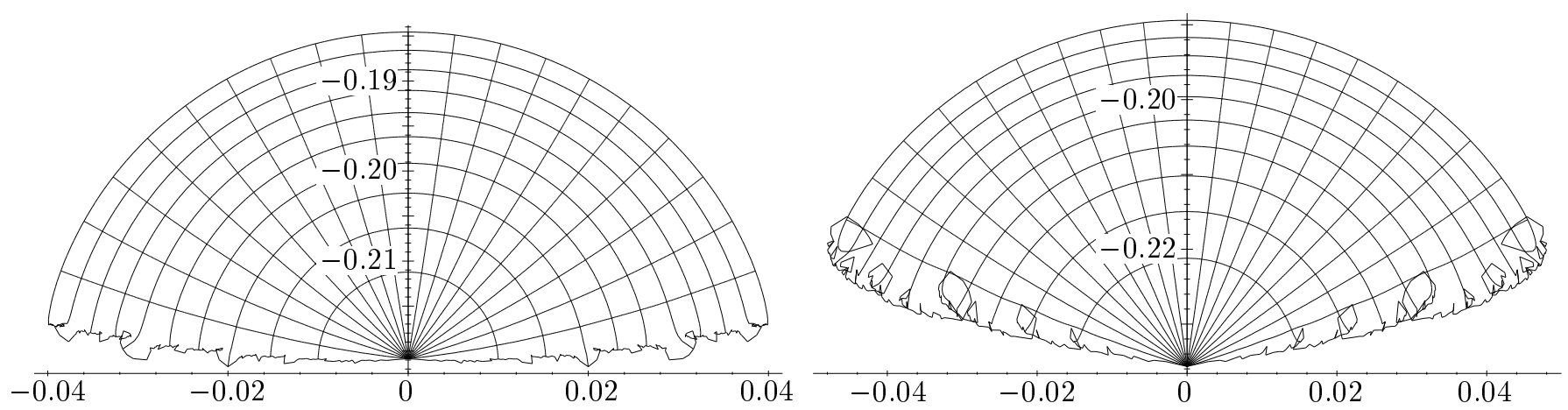

FIGURE 8. The same as Figure 6, except that the i.f.s. used has contraction ratio 0.51 (left) and 0.49 (right). 
$K$, the pentagasket, shown in Figure 9. In Figure 10 we display the analogous image to Figure 6 for the self-similar measure with all $p_{j}=\frac{1}{5}$ associated with this i.f.s.

\section{ACKNOWLEDGMENTS}

We are grateful to Clifford Earle, John Hubbard and John Smillie for helpful discussions.

\section{REFERENCES}

[Carleson 1967] L. Carleson, Selected problems on exceptional sets, Van Nostrand Mathematical Studies 13, Van Nostrand, Princeton, NJ and Toronto, 1967.

[Garnett 1972] J. Garnett, Analytic capacity and measure, Lecture Notes in Math. 297, Springer, Berlin, 1972.

[Hutchinson 1981] J. E. Hutchinson, "Fractals and selfsimilarity", Indiana Univ. Math. J. 30:5 (1981), 713747.
[Mattila 1995] P. Mattila, Geometry of sets and measures in Euclidean spaces: fractals and rectifiability, Cambridge Studies in Advanced Mathematics 44, Cambridge University Press, Cambridge, 1995.

[Mattila and Melnikov 1994] P. Mattila and M. S. Melnikov, "Existence and weak-type inequalities for Cauchy integrals of general measures on rectifiable curves and sets", Proc. Amer. Math. Soc. 120:1 (1994), 143-149.

[Schief 1994] A. Schief, "Separation properties for selfsimilar sets", Proc. Amer. Math. Soc. 122:1 (1994), $111-115$.

[Strichartz 1990] R. S. Strichartz, "Self-similar measures and their Fourier transforms, I", Indiana Univ. Math. J. 39:3 (1990), 797-817.

[Strichartz et al. 1995] R. S. Strichartz, A. Taylor, and T. Zhang, "Densities of self-similar measures on the line", Experiment. Math. 4:2 (1995), 101-128.

[Verdera 1992] J. Verdera, "A weak type inequality for Cauchy transforms of finite measures", Publicacions Matemàtiques 36:2B (1992), 1029-1034 (1993).

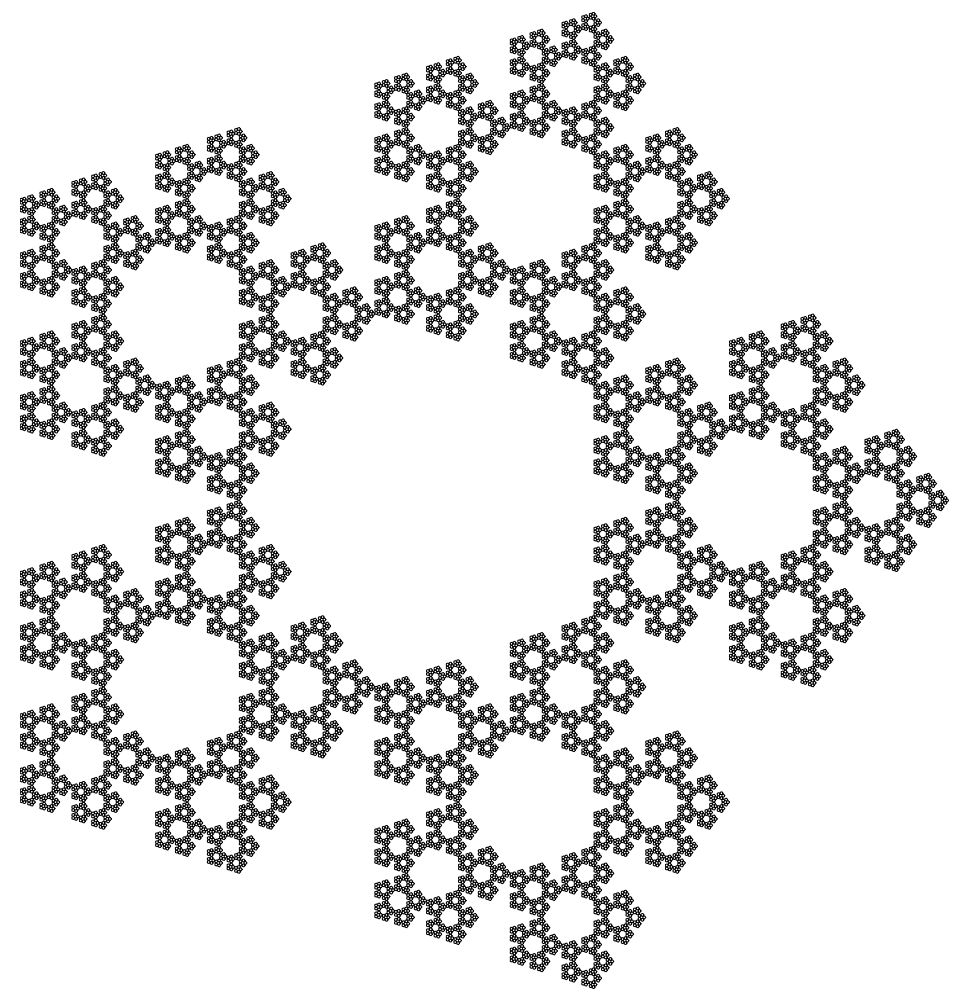

FIGURE 9. The pentagasket (courtesy of Kyallee Dalrymple). 


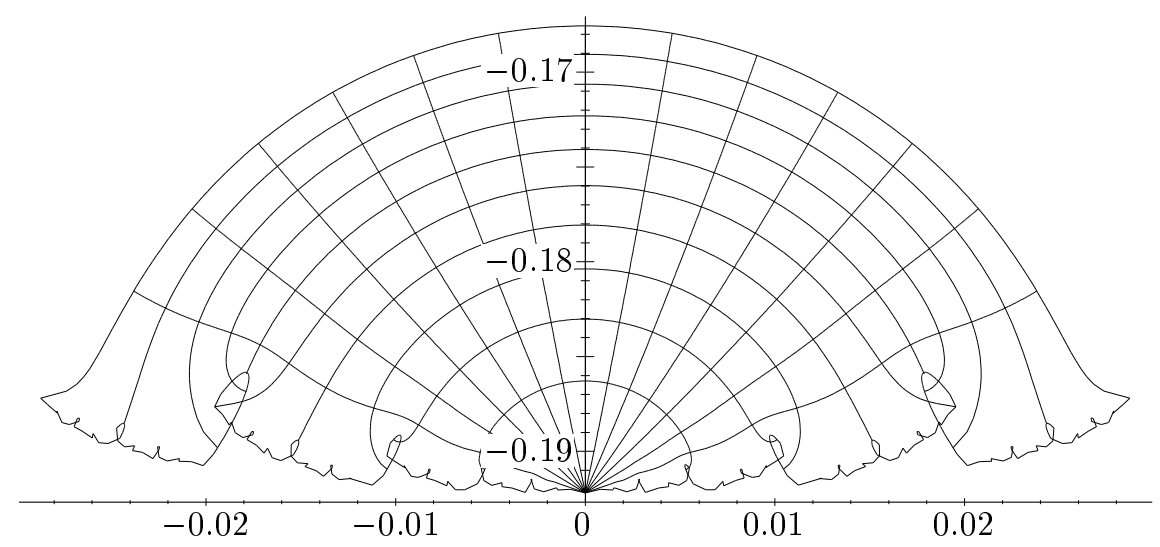

FIGURE 10. The image under $F$ of the exterior portion of a dartboard centered at the corner 1 of the pentagasket.

Received April 29, 1997; accepted in revised form October 2, 1997

John-Peter Lund, Mathematics Department, University of California, Berkeley, CA, 94720, United States (jpl@uclink4.berkeley.edu)

Robert S. Strichartz, Mathematics Department, White Hall, Cornell University, Ithaca, NY 14853 (str@math.cornell.edu)

Jade P. Vinson, Mathematics Department, Washington University, St. Louis, MO 63130

Current address: Mathematics Department, Princeton University, Princeton, NJ 08544

(jpvinson@math.princeton.edu) 